NASA Technical Memorandum 84304

NASA-TM-84304 19830011249

\title{
Accelerating an Iterative Process by Explicit Annihilation
}

Dennis C. Jespersen and Pieter G. Buning

\section{LIBRARY COPY \\ FEB 161983 \\ LANGLEY RESEARCH CENTER \\ LIBRARY, NASA \\ HAMPTON, VIRGINIA}




\section{Accelerating an Iterative Process by Explicit Annihilation}

Dennis C. Jespersen, Informatics General Corporation, Palo Alto, California

Pieter G. Buning, Ames Research Center, Moffett Field, California

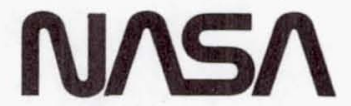

National Aeronautics and

Space Administration

Ames Research Center

Moffett Field, California 94035 


\title{
Accelerating an Iterative Process by Explieit Annihilation
}

\author{
Dennis C. Jespersen* and Pieter G. Buning
}

\author{
Ames Research Center
}

\begin{abstract}
Summary
A slowly convergent stationary iterative process can be accelerated by explicitly annihilating (i.e., eliminating) the dominant eigenvector component of the error. The dominant eigenvalue or complex pair of eigenvalues can be estimated from the solution during the iteration. The corresponding eigenvector or complex pair of eigenvectors can then be annihilated by applying an explicit Richardson process over the basic iterative method. This can be done entirely in real arithmetic by analytically combining the complex conjugate annihilation steps. We illustrate by applying the technique to an implicit algorithm for the calculation of two-dimensional steady transonic flow over a circular cylinder using the equations of compressible inviscid gas dynamics. This demonstrates the use of explicit annihilation on a nonlinear problem.
\end{abstract}

\section{Introduction}

A great deal of work is being done to develop efficient algorithms for steady-state problems in computational fluid dy namics. The most widely used class of algorithms for steady problems consists of time-like methods that are marched to a steady state. Our purpose here is to point out a simple and easily applied technique from linear algebra which can in some cases produce a remarkable speedup in algorithms that march to the steady state. The idea may be briefly summarized as follows. In the later stages of the marching algorithm, the iteration may be behaving linearly. If so, one can estimate the dominant eigenvalue of the underlying iteration matrix and "annihilate" the eigenvector corresponding to this eigenvalue by a special iteration step. Even when the eigenvalue and eigenvector are complex, all the computations can be performed in real arithmetic.

Some notation is developed and some facts from linear algebra are reviewed in the next section. A particular algorithm for the Euler equations is presented in the third section, and an application of annihilation to steady transonic flow over a circular cylinder is described in the final section. We wish to emphasize that the application of annihilation is not restricted to the particular marching algorithm then we use for illustrative purposes.

We extend our thanks to Harvard Lomax for introducing us to the idea of eigenvector annihilation.

\section{Review of Linear Algebra}

In this section we establish our notation and review some elementary facts from numerical linear algebra. To begin, consider the matrix problem $A x=b$ where $A$ is nonsingular. Let $A=M-N$

"Member, Professional Staff, Informatics General Corporation, Palo Alto, California. Work supported by NASA Contract NAS2-8891. 
be a splitting of $A$, where $M$ is nonsingular, and consider the iteration

$$
\begin{aligned}
M x^{n+1} & =N x^{n}+b, \quad n \geq 0, \quad x^{0} \text { given, } \\
\text { or } M \Delta x^{n} & =-A x^{n}+b, \\
\text { and } x^{n+1} & :=x^{n}+\Delta x^{n} .
\end{aligned}
$$

(The notation " $a:=b$ " is used here to mean that $a$ is defined as $b$.)

Let $x^{*}$ satisfy $A x^{*}=b$, and denote the error by $e^{n}:=x^{*}-x^{n}$. Then $M e^{n+1}=N e^{n}$, and so the iteration converges if and only if the spectral radius of $M^{-1} N$ is less than $1, \rho\left(M^{-1} N\right)<1$.

Suppose now that $M^{-1} N$ is diagonalizable with eigenvalues $\lambda_{i}$ and corresponding eigenvectors $v_{i}$. Take $\left|\lambda_{1}\right| \geq\left|\lambda_{2}\right| \geq \ldots \geq\left|\lambda_{m}\right|$, and write $e^{n}=\sum_{i=1}^{m} \alpha_{i} v_{i}$. Then we clearly have

$$
\begin{aligned}
e^{n+k} & =\sum_{i=1}^{m} \alpha_{i} \lambda_{i}^{k} v_{i} \\
& =\lambda_{1}^{k}\left(\alpha_{1} v_{1}+\sum_{i=2}^{m} \alpha_{i}\left(\lambda_{i} / \lambda_{1}\right)^{k} v_{i}\right) .
\end{aligned}
$$

Evidently the component of the error in the direction of $v_{1}$ is the slowest decreasing component (if $\left.\left|\lambda_{1}\right|>\left|\lambda_{2}\right|\right)$.

The component of the error in any direction $v_{j}$ can be annihilated as follows. After computing $\Delta x^{n+k}$, define $x^{n+k+1}:=x^{n+k}+\sigma \Delta x^{n+k}$, where $\sigma$ is as yet arbitrary. (We call this a Richardson step with parameter $\sigma$.) Then we have

$$
\begin{aligned}
e^{n+k+1} & =e^{n+k}-\sigma \Delta x^{n+k} \\
& =e^{n+k}-\sigma M^{-1}\left(-A x^{n+k}+b\right) \\
& =e^{n+k}-\sigma M^{-1} A e^{n+k} \\
& =\left[I-\sigma M^{-1}(M-N)\right] e^{n+k} \\
& =\left[(1-\sigma) I+\sigma M^{-1} N\right] \sum_{i} \alpha_{i} \lambda_{i}^{k} v_{i} \\
& =\sum_{i} \alpha_{i} \lambda_{i}^{k}\left[(1-\sigma)+\sigma \lambda_{i}\right] v_{i} .
\end{aligned}
$$

Thus if we choose $\sigma=1 /\left(1-\lambda_{j}\right)$, the component of $e^{n+k+1}$ in the direction of $v_{j}$ is zero. Any component $v_{j}$ can in theory be annihilated, but in practice the important component is the eigenvector $v_{1}$ associated with the dominant eigenvalue $\lambda_{1}$.

Of course, we do not know $\lambda_{1}$, but we can use the sequence $\Delta x^{n}$ to estimate it, since $\Delta x^{n+1}=$ $M^{-1} N \Delta x^{n}$. Estimating $\lambda_{1}$ from the sequence $\Delta x^{n}$ is simply using the power method to find the dominant eigenvalue of a matrix. Thus we estimate $\lambda_{1}$ by $\lambda_{1} \approx\left(\Delta x^{n+k}\right)_{r} /\left(\Delta x^{n+k-1}\right)_{r}$ for some appropriate $r^{t h}$ component of the update $\Delta x$. (The residual $r^{n+k}:=-A x^{n+k}+b$ could also be used for the estimation, since $r^{n+k}=A e^{n+k}$.) In summary, we plan to estimate $\lambda_{1}$ from the sequence $\Delta x^{n}$ and take a Richardson step with parameter $\sigma=1 /\left(1-\lambda_{1}\right)$.

The general condition for stability of the Richardson step with parameter $\sigma$ is $\left|1-\sigma\left(1-\lambda_{i}\right)\right| \leq$ 1 for all eigenvalues $\lambda_{i}$ of $M^{-1} N$. This ensures that no component of the error is amplified by the Richardson step. It may well happen that the Richardson step we propose is "unstable" in the sense that some components of the error are magnified. We note, however, that in the later stages of an iteration the subdominant components of the error are liable to be so small that a certain amplification of them is acceptable when accompanied by a large decrease in the 
dominant component of the error. Also, further ordinary steps will rapidly reduce these subdominant components again. To be more specific, suppose $e^{n+k}=\alpha_{1} v_{1}+\epsilon v_{i}, i \neq 1$. Then we easily find

$$
\begin{aligned}
e^{n+k+1} & =e^{n+k}-\sigma M^{-1} A e^{n+k} \\
& =\alpha_{1}\left(1-\sigma+\sigma \lambda_{1}\right) v_{1}+\epsilon\left(1-\sigma+\sigma \lambda_{i}\right) v_{i} \\
& =\epsilon \frac{\lambda_{i}-\lambda_{1}}{1-\lambda_{1}} v_{i}, \quad \text { if } \sigma=1 /\left(1-\lambda_{1}\right) .
\end{aligned}
$$

Thus, the $v_{i}$ error component is multiplied by a factor of $\left(\lambda_{i}-\lambda_{1}\right) /\left(1-\lambda_{1}\right)$. We expect $\lambda_{1}$ to be close to 1 , so this factor could be large, but the factor of $\epsilon$ will compensate if it is small enough.

We remark here that in our application, $A$ is not constant, but $A=A\left(x^{n}\right)$. Nevertheless, the assumption of linear behavior will be approximately satisfied in the later stages of the iteration. We will not attempt annihilation unless the iteration seems to be behaving linearly. An empirical criterion for determining this is described in the last section.

We will now show how annihilation can proceed in real arithmetic even when the dominant eigenvalue is complex. It is quite possible that the dominant eigenvalue could be complex, and thus (since we will assume that $A$ is real) that there is a dominant pair of complex conjugate eigenvalues, say $\lambda_{2}=\bar{\lambda}_{1}$, and $\left|\lambda_{1}\right|=\left|\lambda_{2}\right|>\left|\lambda_{3}\right| \geq \ldots \geq\left|\lambda_{m}\right|$. Then if we write $\Delta x^{n}=\sum_{i=1}^{m} \beta_{i} v_{i}$, we see

$$
\Delta x^{n+k-p}=\lambda_{1}^{k-p} \beta_{1} v_{1}+\lambda_{2}^{k-p} \beta_{2} v_{2}+O\left(\left|\lambda_{3}\right|^{k-p}\right)
$$

for $p=0,1,2$. Thus, for any real $c$ and $d$,

$$
\begin{gathered}
\Delta x^{n+k}+c \Delta x^{n+k-1}+d \Delta x^{n+k-2}=\lambda_{1}^{k-2} \beta_{1}\left(\lambda_{1}^{2}+c \lambda_{1}+d\right) v_{1} \\
+\lambda_{2}^{k-2} \beta_{2}\left(\lambda_{2}^{2}+c \lambda_{2}+d\right) v_{2}+O\left(\left|\lambda_{3}\right|^{k-2}\right) .
\end{gathered}
$$

The coefficients of $v_{1}$ and $v_{2}$ will vanish if $c=-2 \operatorname{Re} \lambda_{1}$ and $d=\left|\lambda_{1}\right|^{2}$, for then $\lambda^{2}+c \lambda+d=$ $\left(\lambda-\lambda_{1}\right)\left(\lambda-\lambda_{2}\right)$.

In practice, we can pick indices $i \neq j$ and find $c$ and $d$ such that

$$
\begin{aligned}
\left(\Delta x^{n+k}\right)_{i}+c\left(\Delta x^{n+k-1}\right)_{i}+d\left(\Delta x^{n+k-2}\right)_{i} & =0 \\
\left(\Delta x^{n+k}\right)_{j}+c\left(\Delta x^{n+k-1}\right)_{j}+d\left(\Delta x^{n+k-2}\right)_{j} & =0 .
\end{aligned}
$$

This is a linear system for $c$ and $d$. Once $c$ and $d$ are known, $\lambda_{1}=-c / 2+i \sqrt{d-c^{2} / 4}$ and two Richardson steps can be performed by defining $\sigma:=1 /\left(1-\lambda_{1}\right)$ and putting

$$
\begin{aligned}
& x^{n+k+1}:=x^{n+k}+\sigma \Delta x^{n+k} \\
& x^{n+k+2}:=x^{n+k+1}+\bar{\sigma} \Delta x^{n+k+1},
\end{aligned}
$$

where $\Delta x$ is still given by one step of the original iterative scheme, namely, $\Delta x^{n+k}:=$ $M^{-1}\left(-A x^{n+k}+b\right)$ and $\Delta x^{n+k+1}:=M^{-1}\left(-A x^{n+k+1}+b\right)$. By the calculations done previously, this will eliminate the $v_{1}$ and $v_{2}$ components of the error. Furthermore, there is no need for complex arithmetic, for the computation can be reorganized as follows:

$$
\begin{aligned}
M\left(x^{n+k+2}-x^{n+k}\right)= & M\left(\bar{\sigma} \Delta x^{n+k+1}+\sigma \Delta x^{n+k}\right) \\
= & \bar{\sigma}\left(-A x^{n+k+1}+b\right)+\sigma\left(-A x^{n+k}+b\right) \\
= & \bar{\sigma}\left[-A\left(x^{n+k}+\sigma \Delta x^{n+k}\right)\right] \bar{\sigma} b-\sigma A x^{n+k}+\sigma b \\
= & -(\sigma+\bar{\sigma}) A x^{n+k}+(\sigma+\bar{\sigma}) b \\
& \quad-|\sigma|^{2} A M^{-1}\left(-A x^{n+k}+b\right) \\
= & 2 \operatorname{Re} \sigma r^{n+k}-|\sigma|^{2} A M^{-1} r^{n+k} .
\end{aligned}
$$


Thus, two complex Richardson steps can be performed entirely in real arithmetic, at a cost of (1) solving two linear systems with coefficient matrix $M$ and (2) performing one matrix-vector multiplication with coefficient matrix $A$. This is the same as the cost of two steps of the basic iteration process. As for stability, a component of $e^{n+k}$ in the direction of the vector $v_{j}, j>2$, is multiplied by the factor

$$
\frac{\left(\lambda_{j}-\lambda_{1}\right)\left(\lambda_{j}-\bar{\lambda}_{1}\right)}{\left(1-\lambda_{1}\right)\left(1-\bar{\lambda}_{1}\right)} .
$$

Again, this factor could be large, but we expect to apply the annihilation in the later stage of the iteration when the coefficients of $v_{j}$ are very small and some amplification would be tolerable.

We might remark that the presence of a dominant complex conjugate pair of eigenvalues is signaled by oscillations in the norm of the residual. For example, if $r^{1}=\alpha_{1} A v_{1}+\alpha_{2} A v_{2}=2 \operatorname{Re}\left(\alpha_{1} A v_{1}\right)$, then writing $\lambda_{1}=\rho e^{i \theta}$ we see that in any norm

$$
\begin{aligned}
\rho^{-k}\left\|r^{k}\right\| & =\left\|\alpha_{1} e^{i k \theta} A v_{1}+\bar{\alpha}_{1} e^{-i k \theta} A \bar{v}_{1}\right\| \\
& =\left\|2 \operatorname{Re}\left(\alpha_{1} e^{i k \theta} A v_{1}\right)\right\| .
\end{aligned}
$$

Thus, the vector $\alpha_{1} A v_{1}$ is rotated through an angle $k \theta$ and projected on the real axis, which will give oscillations. Furthermore, if $\theta=\pi p / q$ in lowest terms, then the period of the oscillation will be $q$. Examples of oscillatory decreasing residuals (which signal the presence of a dominant complex conjugate eigenvalue pair) can be found in references $1-3$.

We close this section with some remarks on the relation of annihilation to previous work. It is easy to show that in the case of a scalar sequence $\left(x^{0}, x^{1}, \ldots, x^{n}\right)$, our "annihilation" step is, exactly, an Aitken $\delta^{2}$-extrapolation step. Wilkinson (ref. 4) discusses the application of the Aitken $\delta^{2}$ technique to the power method for estimating eigenvalues and eigenvectors, where $x^{n}$ is a vector. The formulas there are different from the ones we have used: in Wilkinson's formulation the Aitken $\delta^{2}$ acceleration step is applied to each component of the vector separately (in effect, an "eigenvalue" is assumed for each separate component of the sequence of vectors), whereas in our formulation a single eigenvalue is estimated and used for all components of the vector. We are unable to say which strategy is better. Furthermore, in Wilkinson's formulation it is unclear how to handle the case of a dominant complex conjugate pair of eigenvalues (it is shown how to estimate the eigenvalues as roots of a quadratic equation, but the acceleration of the convergence of the vectors is not discussed). The idea of annihilation is elementary but appears to have been neglected. Lyusternik (ref. 5) considered a procedure that amounts to annihilation in the case in which the eigenvalues are all real, but he did not discuss the complex case. Hyman and Manteuffel (ref. 6) described an algorithm very similar to annihilation for accelerating slowly convergent iterative methods. In their algorithm, eigenvalue estimates are obtained via a Krylov sequence technique.

Many authors have been concerned with the harder problem of optimizing relaxation schemes (getting all the eigenvalues of the iteration matrix as far inside the unit circle as possible). An adaptive procedure for minimizing the norm of the iteration matrix was presented by Manteuffel (ref. 7); he estimated complex eigenvalues by the same technique we use above. To reiterate our point of view: we do not ask for the "optimum" method in some class of methods, nor do we insist that our annihilation steps be stable. We simply estimate eigenvalues and perform annihilation. Moreover, we are willing to accept amplification of some error components in the annihilation step; these components will be rapidly reduced anyway in subsequent normal iteration steps. 


\section{An Iterative Method for the Euler Equations}

In this section we will describe an iterative method for some steady-state problems in fluid dynamics. The governing equations are the steady two-dimensional Euler equations of compressible inviscid gas dynamics, which express the conservation of mass, momentum, and energy. They can be written in the form

$$
\frac{\partial E(Q)}{\partial x}+\frac{\partial F(Q)}{\partial y}=0
$$

where $Q$ is the four-vector $Q=(\rho, \rho u, \rho v, e)^{T}$. Here $\rho$ is density, $u$ and $v$ are Cartesian velocity components, and $e$ is total energy per unit volume. The functions $E, F: D \subset \mathbf{R}^{4} \rightarrow \mathbf{R}^{4}$ are nonlinear functions given by

$$
\begin{aligned}
& E(Q)=\left(\rho u, \rho u^{2}+p, \rho u v, u(e+p)\right)^{T} \\
& F(Q)=\left(\rho v, \rho u v, \rho v^{2}+p, v(e+p)\right)^{T}
\end{aligned}
$$

The pressure $p$ is defined by $p=[\gamma-1)\left(e-\frac{1}{2} \rho\left(u^{2}+v^{2}\right)\right]$ where $\gamma$ is a constant. Appropriate boundary conditions must be adjoined to the differential equation to complete the specification of the problem. It is not important for our purposes what these boundary conditions are, so we will not discuss them further. In order to handle a curved geometry, we map from the physical $(x, y)$ domain to a "computational" $(\xi, \eta)$ domain; the computational domain is usually taken to be a rectangle, for ease in differencing. In the $(\xi, \eta)$ coordinates, the transformed equations retain the strong conservation law form of equation (11) (ref. 8), becoming

$$
\frac{\partial \hat{E}(\hat{Q})}{\partial \xi}+\frac{\partial \hat{F}(\hat{Q})}{\partial \eta}=0
$$

Iterative procedures for solving the steady-state equations (12) often take as their starting point the unsteady equations

$$
\frac{\partial \hat{Q}}{\partial t}+\frac{\partial \hat{E}(\hat{Q})}{\partial \xi}+\frac{\partial \hat{F}(\hat{Q})}{\partial \eta}=0 .
$$

If we choose Euler implicit differencing in time (hoping for good stability properties) we get the iteration

$$
\hat{Q}^{n+1}=\hat{Q}^{n}-h\left(\delta_{\xi} \hat{E}^{n+1}+\delta_{\eta} \hat{F}^{n+1}\right),
$$

where $\delta_{\xi}, \delta_{\eta}$ are spatial difference operators, $h:=\Delta t$, and $n$ denotes a time level. To avoid iteration on the nonlinear flux terms, $\hat{E}^{n+1}$ and $\hat{F}^{n+1}$ on the right-hand side of equation (14) are expanded about values at level $n$ using a Taylor series

$$
\begin{aligned}
\hat{E}^{n+1} & =\hat{E}^{n}+\left.\frac{\partial \hat{E}}{\partial \hat{Q}}\right|^{n} \Delta \hat{Q}^{n}+O\left(\left(\Delta \hat{Q}^{n}\right)^{2}\right) \\
\Delta \hat{Q}^{n} & =\hat{Q}^{n+1}-\hat{Q}^{n} .
\end{aligned}
$$

The locally linearized form of equation (14) can then be written in "delta" form as

$$
\left[I+h\left(\delta_{\xi} \hat{A}^{n}+\delta_{\eta} \hat{B}^{n}\right)\right] \Delta \hat{Q}^{n}=-h\left(\delta_{\xi} \hat{E}^{n}+\delta_{\eta} \hat{F}^{n}\right),
$$


where $\hat{A}^{n}$ and $\hat{B}^{n}$ are the Jacobian matrices $\partial \hat{E}^{n} / \partial \hat{Q}^{n}$ and $\partial \hat{F}^{n} / \partial \hat{Q}^{n}$, respectively.

Each step of equation (16) involves the inversion of a large block-banded matrix, with halfbandwidth equal to the number of points in one direction of the mesh. The amount of work required for this is unacceptably large; instead the matrix is approximately factored (ref. 9) For example, if $\delta_{\xi}$ and $\delta_{\eta}$ were three-point central difference operators, the left-hand side of $(16)$ might be factored via

$$
\left[I+h\left(\delta_{\xi} \hat{A}^{n}+\delta_{\eta} \hat{B}^{n}\right)\right]=\left(I+h \delta_{\xi} \hat{A}^{n}\right)\left(I+h \delta_{\eta} \hat{B}^{n}\right)+O\left(h^{2}\right)
$$

as the product of two block-tridiagonal matrices.

The calculations we will show come from an algorithm that uses flux-vector splitting (refs. 10, 11 ), which is based on separating positive and negative characteristic directions to allow the use of one-sided spatial difference operators. The Euler equations (13) are hyperbolic, and $\hat{E}=\hat{A} \hat{Q}$ can be decomposed as

$$
\begin{aligned}
\hat{E} & =\hat{A} \hat{Q}=X \Lambda X^{-1} \hat{Q} \\
& =X\left(\Lambda^{+}+\Lambda^{-}\right) X^{-1} \hat{Q} \\
& =\left(\hat{A}^{+}+\hat{A}^{-}\right) \hat{Q} \\
& =\hat{E}^{+}+\hat{E}^{-},
\end{aligned}
$$

where $\Lambda$ is the diagonal matrix of eigenvalues of $\hat{A}$, and $\Lambda^{+}$and $\Lambda^{-}$are the positive and negative parts of $\Lambda$, respectively. $\hat{F}$ can be similarly decomposed. Defining $\tilde{A}^{+}:=\partial \hat{E}^{+} / \partial \hat{Q}$, and similarly for $\tilde{A}^{-}, \tilde{B}^{+}$, and $\tilde{B}^{-}$, the unfactored scheme is

$$
\begin{array}{r}
{\left[I+h\left(\delta_{\xi}^{b} \tilde{A}^{+}+\delta_{\xi}^{f} \tilde{A}^{-}+\delta_{\eta}^{b} \tilde{B}^{+}+\delta_{\eta}^{f} \tilde{B}^{-}\right)^{n}\right] \Delta \hat{Q}^{n}} \\
=-h\left(\delta_{\xi}^{b} \hat{E}^{+}+\delta_{\xi}^{f} \hat{E}^{-}+\delta_{\eta}^{b} \hat{F}^{+}+\delta_{\eta}^{f} \hat{F}^{-}\right)^{n},
\end{array}
$$

where $\delta^{b}$ and $\delta^{f}$ are backward and forward spatial difference operators. On the left-hand side, the forward difference operators are separated from the backward, resulting in an approximate factorization into lower and upper block-triangular matrices. First-order spatial differences $\Delta^{b}$ and $\Delta^{f}$ are used on the left-hand side and second-order differences $\delta^{b}$ and $\delta^{f}$ are used on the right-hand side, to give second-order accuracy in the steady state. We have, for instance,

$$
\begin{aligned}
\Delta_{\xi}^{b} \hat{A}_{i} & :=\hat{A}_{i}-\hat{A}_{i-1} \\
\delta_{\xi}^{b} \hat{E}_{i} & :=\left(3 \hat{E}_{i}-4 \hat{E}_{i-1}+\hat{E}_{i-2}\right) / 2 .
\end{aligned}
$$

The full scheme is thus

$$
\begin{gathered}
{\left[I+h\left(\Delta_{\xi}^{b} \tilde{A}^{+}+\Delta_{\eta}^{b} \tilde{B}^{+}\right)^{n}\right]\left[I+h\left(\Delta_{\xi}^{f} \tilde{A}^{-}+\Delta_{\eta}^{f} \tilde{B}^{-}\right)^{n}\right] \Delta \hat{Q}^{n}} \\
=-h\left(\delta_{\xi}^{b} \hat{E}^{+}+\delta_{\xi}^{\delta} \hat{E}^{-}+\delta_{\eta}^{b} \hat{F}^{+}+\delta_{\eta}^{f} \hat{F}^{-}\right)^{n} .
\end{gathered}
$$

In the notation discussed in the preceding section,

$$
\begin{aligned}
M & =\left[I+h\left(\Delta_{\xi}^{b} \tilde{A}^{+}+\Delta_{\eta}^{b} \tilde{B}^{+}\right)^{n}\right]\left[I+h\left(\Delta_{\xi}^{\rho} \tilde{A}^{-}+\Delta_{\eta}^{f} \tilde{B}^{-}\right)^{n}\right] \\
A & =h\left(\delta_{\xi}^{b} \tilde{A}^{+}+\delta_{\xi}^{\rho} \tilde{A}^{-}+\delta_{\eta}^{b} \tilde{B}^{+}+\delta_{\eta}^{\rho} \tilde{B}^{-}\right)^{n} .
\end{aligned}
$$

It is clear that $A$ is not a constant matrix, but if we wait until the later stages of the iteration, $A$ will be changing very slowly, and we should be able to apply annihilation with a fair degree of success. 


\section{Application to Steady Transonic Flow}

The annihilation idea has been applied to the numerical method given in the preceding section for the Euler equations. In this section we will describe the physical problem, show how to apply annihilation, and give some results showing the success of annihilation. We will also mention some questions and problem areas that our experiences have identified.

We performed calculations for flow over a circular cylinder with symmetry imposed between the top and bottom, so that only the top half of the region was calculated. Figure 1 shows the grid used, including the two points below the symmetry line in front of and behind the cylinder used to impose the symmetry condition. At the far-field boundary, 16 diameters away from the cylinder, all flow variables are fixed at their free-stream values. Boundary conditions at the body consist of setting the normal velocity to zero, taking surface density and tangential velocity from the point above, and calculating pressure from a conservation of momentum relation.

At a free-stream Mach number of 0.5 , a shock forms on the cylinder. A steady solution for this case is shown in figure 2. The shock has introduced rotationality into the flow and caused inviscid flow separation on the back side of the cylinder.

The application of eigenvector annihilation to the iterative method (eq. (20)) requires some ad hoc decisions on how to estimate the dominant eigenvalue pair. The method presented here is one of many strategies that could be envisioned. As described in the second section (Review of Linear Algebra), one component of $\Delta \hat{Q}$ is needed at two points in the field, corresponding to subscripts $i$ and $j$ in equation (6), for three consecutive iteration steps. We used the first component (density) of $\Delta \hat{Q}$. Four grid points were chosen, each a third of the way in from a corner of the computational domain (see fig. 1). The two points closest to the cylinder form one pair, the other two another pair. In this way we can obtain two estimates of the dominant eigenvalue, from different regions in the grid. The two points from each pair are separated in an attempt to minimize any local coupling effects. During each iteration, eigenvalue estimates are made from the two pairs of points. If the real and imaginary components of the estimates differ by less than $5 \%$, based on the modulus of the first estimate, the two estimates are averaged to produce a candidate $\lambda$. Annihilation is performed if this candidate $\lambda$ is within $5 \%$ of the candidate $\lambda$ from the previous iteration. Thus, our criterion for linear behavior involves a consistent eigenvalue estimate from widely separated points in the grid over four iterations.

The estimated eigenvalue is in general complex; hence, two Richardson steps are required. An outline of the steps performed is given below, using the definitions of the matrices $M$ and $A$ from equation (21):

1. $\Delta \hat{Q}^{n}=M\left(\hat{Q}^{n}\right)^{-1} A\left(\hat{Q}^{n}\right) \hat{Q}^{n}$

2. Decide whether to annihilate. If yes,

3. $\hat{Q}^{n+1}=\hat{Q}^{n}+\left[\left|\sigma^{2}\right| /(2 R e \sigma)\right] \Delta \hat{Q}^{n}$

4. Apply boundary conditions to $\hat{Q}^{n+1}$

5. $\Delta \hat{Q}^{n+1}=M\left(\hat{Q}^{n}\right)^{-1} A\left(\hat{Q}^{n}\right) \hat{Q}^{n+1}$

6. $\hat{Q}^{n+2}=\hat{Q}^{n}+2 \operatorname{Re} \sigma \Delta \hat{Q}^{n+1}$

7. Apply boundary conditions to $\hat{Q}^{n+2}$

The result of applying annihilation, using this strategy, to the cylinder problem is shown in figure 3 . In this figure we compare the result of no annihilation with the result of starting the annihilation strategy at $n=500$. In figure 3 annihilation steps were performed at $n=504,540,577,616,670$, $704,869,892$, and 904 . The convergence rate over the last 300 iterations for the curve without 
annihilation was about 0.9958 , whereas for the curve with annihilation the convergence rate was about 0.9881 . We remark that if only one annihilation step is performed, the residual drops sharply but eventually resumes converging at a rate of 0.9958 . This may be due either to error in the estimation of the eigenvalue (so that the dominant eigenvector component of the error was not completely annihilated) or to nonlinear feedback effects stemming from the fact that the iteration process is truly nonlinear.

We close by mentioning some questions and problem areas that have arisen during this work. First, the choice of the strategy used to estimate the eigenvalues-ours was heuristic - is important. Can better ones be devised? In this regard we would like to mention the note by Jones (ref. 12), ho gives a statistical criterion based on a serial correlation coefficient for deciding when to employ the Aitken technique. The application is to a real scalar sequence; it would be useful to have a similar statistical criterion for a trigonometric sequence modulated by a geometrically decaying term, but we are unaware of a serial correlation coefficient in this case.

Second, it is difficult to decide when to annihilation should be started. In figure 4 we show the result of starting the annihilation strategy at $n=0$. In this case there were 19 annihilation steps: at $n=129,168,208,235,268,310,369,476,491,626,648,656,794,896,918,927,934,951$, and 984 . Evidently this strategy was successful, but not much more so than that of waiting until $n=500$ to start annihilating. This may be due to an incorrect estimation of eigenvalues or to the nonlinearity of the process. Third, stability questions arise when performing annihilation. We see in figure 4 some sharp increases in the residual at certain annihilation steps, and annihilation strategies that do not allow these jumps might be preferred. Finally, the annihilation procedure requires an extra (third) level of computer storage beyond that normally needed for the iterative procedure; this may be a problem in cases where extra space is scarce. 


\section{References}

1. Bell, J. B.; Shubin, G. R.; and Solomon, J. M.: Fully Implicit Shock Tracking, in Numerical Boundary Condition Procedures. NASA CP-2201, 1982.

2. Johnson, Gary M.: Multiple-Grid Acceleration of Lax-Wendroff Algorithms. NASA TM-82843, 1982.

3. Jameson, A.; Schmidt, W.; and Turkel, E.: Numerical Solutions of the Euler Equations by Finite Volume Methods Using Runge-Kutta Time-Stepping Schemes. AIAA Paper 81-1259, June 1981.

4. Wilkinson, J. H.: The Algebraic Eigenvalue Problem. Cambridge University Press, 1966, pp. $578 \mathrm{ff}$.

5. Lyusternik, L. A.: Remarks on the Numerical Solution of Boundary Problems for Laplace's Equation and the Calculation of Characteristic Values by the Method of Networks. Trav. Inst. Math. Stekloff, vol. 20, 1947, pp. 49-64 (in Russian).

6. Hyman, J. M.; and Manteuffel, T. A.: Dynamic Acceleration of Nonlinear Iterations. Presented at SIAM 30th Anniversary Meeting, Stanford, California, July 1982.

7. Manteuffel, T. A.: Adaptive Procedure for Estimating Parameters for the Nonsymmetric Tchebychev Iteration. Numerische Mathematik, vol. 31, pp. 183-208, 1978.

8. Viviand, H.: Conservative Forms of Gas Dynamics Equations. La Recherche Aerospatiale, no. 1, Jan.-Feb. 1974 , p. 65.

9. Warming, R. F.; and Beam, R. M.: On the Construction and Application of Implicit Factored Schemes for Conservation Laws. Symposium on Computational Fluid Dynamics, SLAM-AMS Proc. 11, 1978 .

10. Steger, J. L.; and Warming, R. F.: Flux Vector Splitting of the Inviscid Gasdynamic Equations with Application to Finite-Difference Methods. J. Comp. Phys., vol. 40, 1981, pp. 263-293.

11. Buning, P. G.; and Steger, J. L.: Solution of the Two-Dimensional Euler Equations with Generalized Coordinate Transformation Using Flux Vector Splitting. AIAA Paper 82-0971, June 1982 .

12. Jones, B: A Note on Aitken's $\delta^{2}$ Technique. SIGNUM Newsletter, June 1982, p. 23. 
Page intentionally left blank 


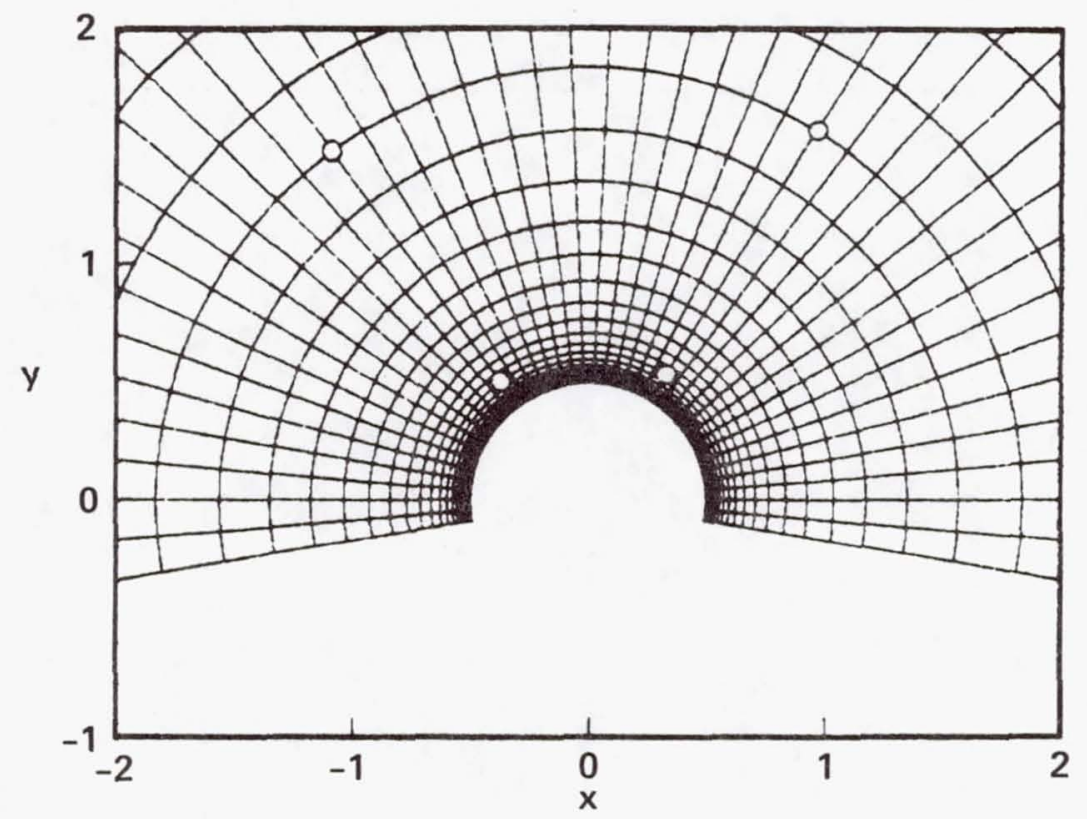

Fig. 1. Exponentially stretched 42-by-31 grid about a circular cylinder, showing points used for eigenvalue estimation.

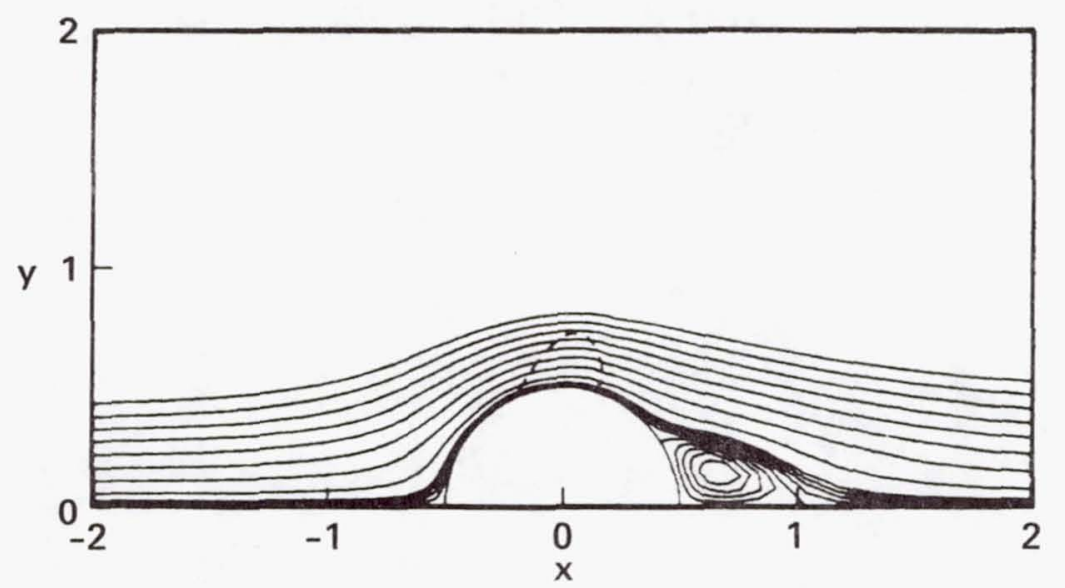

Fig. 2. Streamlines and sonic line for steady flow about a circular cylinder at a free-stream Mach number of 0.5 . 


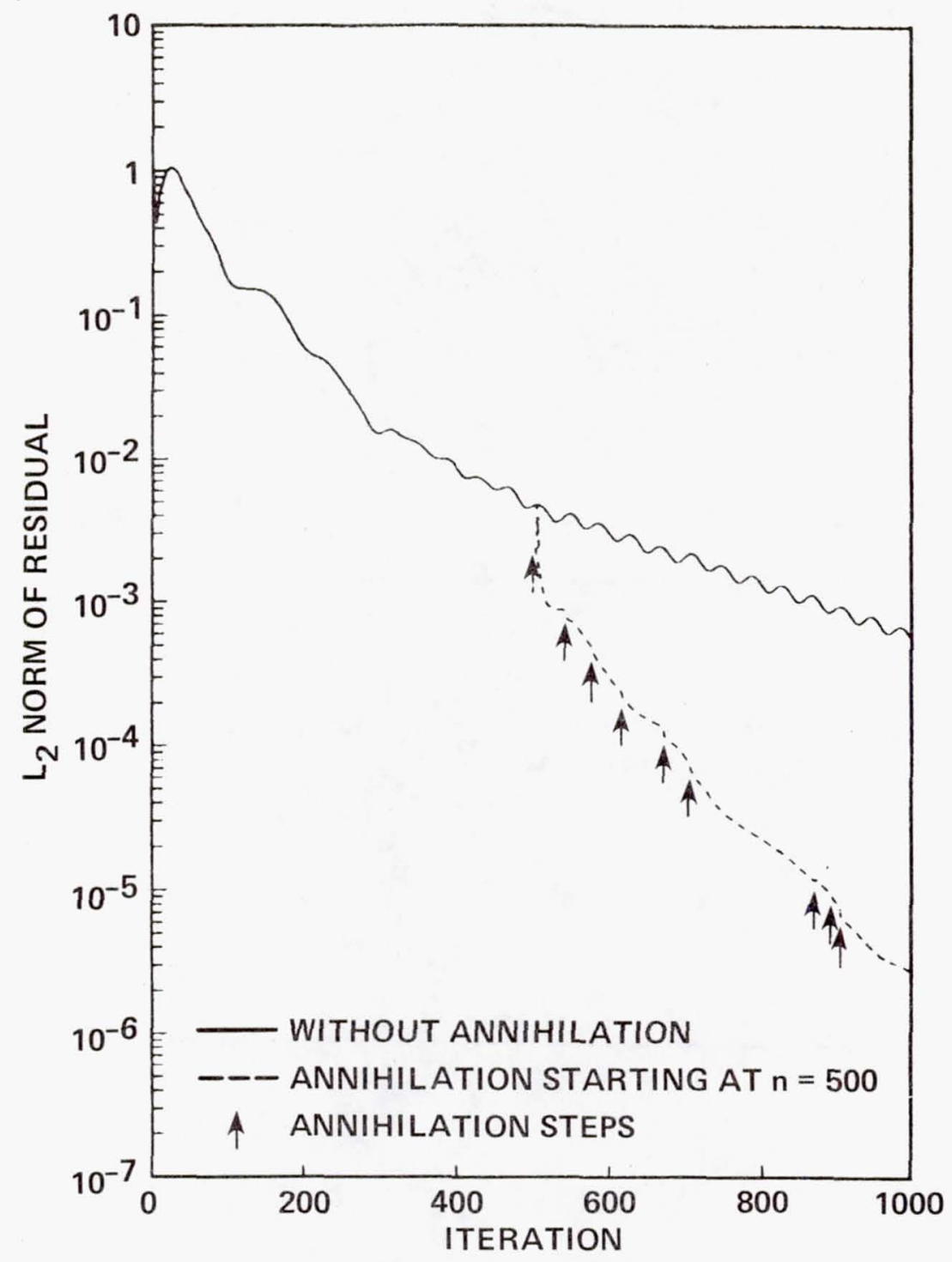

Fig. 3. Comparison of convergence histories without annihilation and with annihilation starting at $n=500$. 


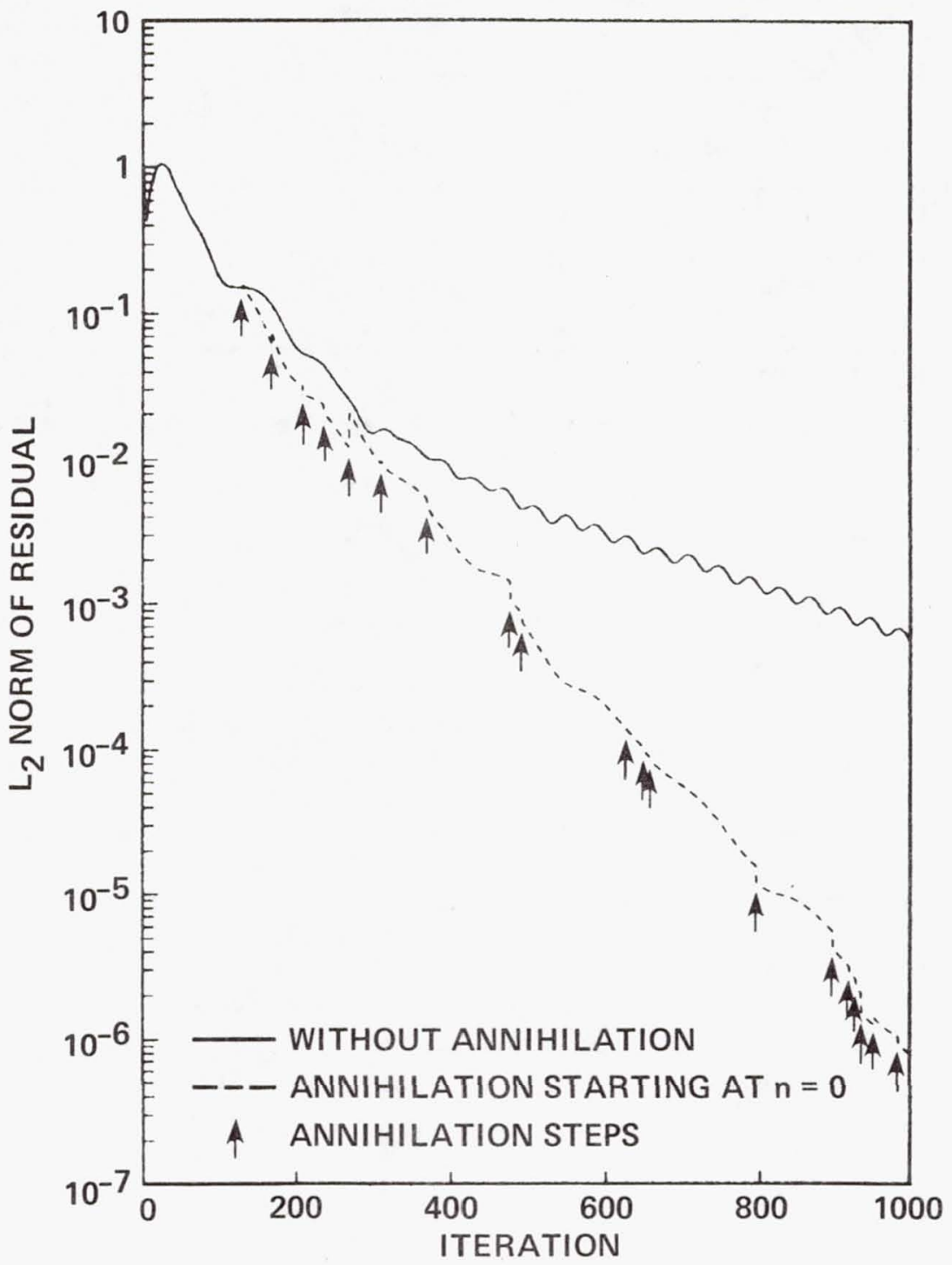

Fig. 4. Convergence history with annihilation starting at $n=0$. 


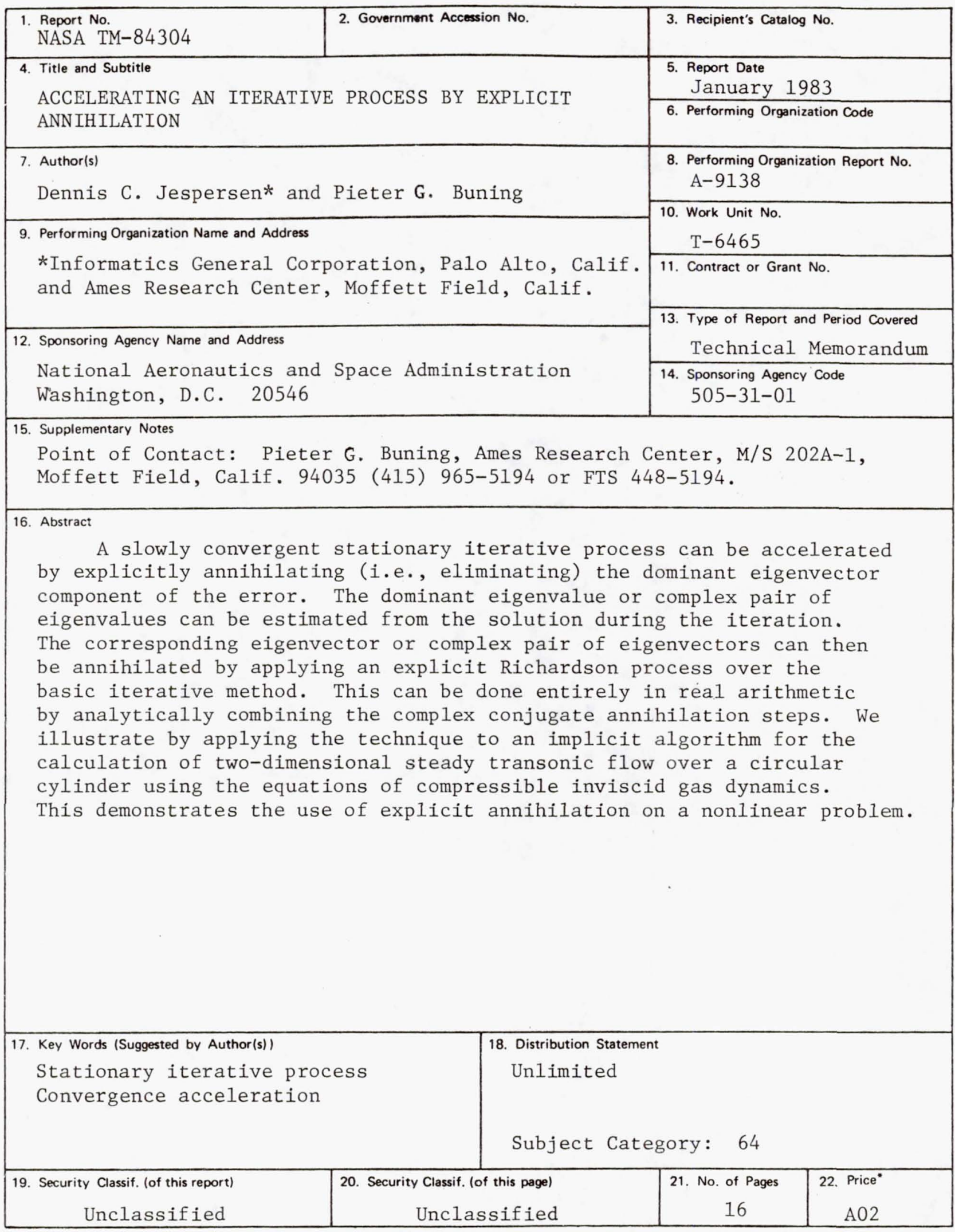

-For sale by the National Technical Information Service, Springfield, Virginia 22161 